\title{
DESIGN AND EVALUATION \\ OF A ROBUST DYNAMIC NEUROCONTROLLER FOR A MULTIVARIABLE AIRCRAFT CONTROL PROBLEM.
}

\author{
T. TROUDET ${ }^{1}$, S. GARG, W. MERRILL \\ Advanced Control Technology Branch \\ NASA Lewis Research Center \\ Cleveland, Ohio 44135
}

\begin{abstract}
.
The design of a dynamic neurocontroller with good robustness properties is presented for a multivariable aircraft control problem. The internal dynamics of the neurocontroller are synthesized by a state estimator feedback loop. The neurocontrol is generated by a multilayer feedforward neural network which is trained through backpropagation to minimize an objective function that is a weighted sum of tracking errors, and control input commands and rates. The neurocontroller exhibits good robustness through stability margins in phase and vehicle output gains. By maintaining performance and stability in the presence of sensor failures in the error loops, the structure of the neurocontroller is also consistent with the classical approach of flight control design.

I. Introduction. There is a synergistic interest by both the Control and the Neural Network communities in designing neural network architectures to solve difficult control problems [1-5]. However, in most of the applications reported in the literature, evaluations of such neurocontrollers have either been limited to the nominal plant model used for the control design, or have pointed to poor robustness properties of the neurocontrol design. The term "robustness" is defined here as maintaining performance and stability in the presence of uncertainties associated with the modelling process.

Since a plant model is only a simplified version of the plant dynamics, an important criterion for the practical design of neurocontrollers is that of robustness. The standard feedforward neural network architecture with plant measurement feedback was investigated in Ref.[5]. The results reported there indicated that this structure does not meet the robustness requirements for flight control systems. Therefore, an important issue in the applicability of neural networks as controllers is that of devising neural architectures with good control robustness properties. The objective of this paper is to address that issue in the context of aerospace vehicle control with special emphasis on piloted flight. Towards that objective, results are presented and discussed for the design/evaluation of a robust neurocontroller to provide independent control of pitch rate anc airspeed responses to pilot command inputs for an integrated airframe/propulsion longitudinal dynamics model of a modern fighter aircraft.

The paper is organized as follows. The vehicle model and the desired closed-loop dynamics are introduced in Section II, and are followed in Section III by the training architecture and the design of the neurocontroller. The nominal performance and the robustness of the dynamic neurocontroller are evaluated and discussed in section IV.

II. Vehicle Model. The vehicle model consists of an integrated state-space representation for a modern fighter aircraft powered by a two-spool turbofan engine and equipped with a two-dimensional thrustvectoring and reversing nozzle. The flight condition used in this application is representative of the STOL (Short Take-off and Landing) approach-to-landing task, with an airspeed of $V_{0}=120 \mathrm{Knots,} \mathrm{a} \mathrm{flight} \mathrm{path}$ angie of $\gamma_{0}=-3 \mathrm{deg}$, and a pitch attitude of $\theta_{0}=7 \mathrm{deg}$. The linearized dynamics of the vehicle model are of the form

$$
\dot{\bar{x}}=A \bar{x}+B \bar{u}_{a}, \quad \bar{z}=C \bar{x} ;
$$

where the state vector is$$
\bar{x}=[u, w, Q, \theta, h, N 2, N 25, P 6, T 41 B]^{T},
$$

$u$ being the aircraft body axis forward velocity (ft/sec), $w$ the aircraft body axis vertical velocity (ft/sec), $Q$ the aircraft pitch rate (rads/sec), $\theta$ the pitch angle (rads), $h$ the altitude (ft), N2 the engine fan speed
\end{abstract}

'T. Troudet is with Sverdrup Technology, Inc., 2001 Aerospace Parkway, Brook Park, Ohio 44142. 
(rpm), N25 the core compressor speed (rpm), P6 the engine mixing plane pressure (psia), and T41B the engine high pressure turbine blade temperature $\left({ }^{\circ} \mathrm{R}\right)$. In Eq.(1), the control input vector is

$$
\left.\bar{u}_{a}=: W F, \delta T V\right]^{T} \text {; }
$$

where $W F$ is the engine main burner fuel flow rate $(\mathrm{lbm} / \mathrm{hr})$, and $\delta T V$ is the nozzle thrust vectoring angle (deg). The vehicle outputs to be controlled are

$$
\bar{z}=[V, Q]^{T},
$$

where $V$ is the aircraft velocity in $\mathrm{ft} / \mathrm{sec}$, and $Q$ is the pitch rate in $\operatorname{deg} / \mathrm{s}$. The system matrices $A, B$, and $C$ are available in Ref.[6]. Open loop analysis indicates that the airframe is statically unstable with a highly unstable pitch mode, and that the response of the controlled outputs $\bar{z}$ is strongly coupled to the control inputs $\bar{u}_{a}$.

The control design objective is to design a control system that provides decoupled command tracking of velocity and pitch rate from pilot control inputs with aircraft responses compatible with Level I handling qualities requirements [7]. The desired response dynamics are selected to be of the form

$$
\dot{\bar{x}}_{m}=A_{m} \bar{x}_{m}+B_{m} \bar{z}_{S E L}, \quad \bar{z}_{c}=C_{m} \bar{x}_{m} ;
$$

with $\bar{z}_{S E L}=\left[V_{S E L}, Q_{S E L}\right]^{T}$ where $V_{S E L}$ is the pilot velocity command in $\mathrm{ft} / \mathrm{s}$, and $Q_{S E L}$ is the pilot longitudinal stick deflection in inches; and $\bar{z}_{c}=\left[V_{c}, Q_{c}\right]^{T}$, where the subscript "c" refers to the ideal response in $V$ and $Q$ with units of $\mathrm{ft} / \mathrm{s}$ and $\mathrm{deg} / \mathrm{s}$ respectively. The system matrices $A_{m}, B_{m}$ and $C_{m}$ are the statespace representation of the ideal response transfer functions listed in Ref.[6].

Actuator modeis were also used in the control design and evaluation. The fuel flow actuator was modelled as

$$
G_{W F}(s)=10 /(s+10) .50 /(s+50) ;
$$

with a maximum fuel flow rate $|W F|_{\max }=10,000 \mathrm{lbm} / \mathrm{hr}$, and a rate limit $|\dot{W} F|_{\max }=20,000 \mathrm{lbm} / \mathrm{hr} / \mathrm{s}$. Note that the fuel flow here corresponds to the perturbation from the trim value for the linear model. In this study, the vaiue $|W F|_{\max }$ is therefore chosen such that the total fuel flow limit will not be exceeded when a perturbation of a magnitude of $|W F|_{\max }$ is commanded. The thrust vectoring actuator is modelled as

$$
G_{\delta T V}(s)=15 /(s+15) ;
$$

with a maximum :arust vector angle $|\delta T V|_{\max }=10 \mathrm{deg}$, and a rate limit $|\delta T V|_{\max }=20 \mathrm{deg} / \mathrm{s}$. As a result, nonlinearities appear in the control design and evaluation in the form of actuator position and rate limits.

III. Design of a Dynamic Neurocontroller. The possibility of designing a neurocontroller that provides a satisfactory trade-off between tracking performance, control effort, and control rates, was demonstrated in Ref.[5]. The neurocontroller designed in [5] was a feedforward neural network having as inputs the vehicle outputs, the tracking errors, the error rates, and the error integrals. This neurocontroller will be referred to in this work as a static neurocontroller. It achieved a nominal performance comparable to that of a baseline $H_{\infty}$-based controller designed for the same command tracking problem. However, the $H_{\infty}$ based controller was significantly more robust than the static neurocontroller in the presence of time-delay and error loop failures.

A comparison of the two controllers as in Ref. $\left[5\right.$, showed that the $H_{\infty}$-based controller had dynamics that are different from the PID (Proportional+Integral+Derivative) structure built in the static neurocontroller. The $H_{\infty}$-based control synthesis automatically generates the controller dynamics that are necessary to achieve the desired performance and robustness. In order to enhance the neurocontrol robustness, it is therefore proposed to investigate procedures by which such automatic synthesis of the desired controller dynamics can be achieved by a neurocontrol design.

For most modern multivariable control design techniques, the controller has an internal structure which consists of a state feedback regulator together with a state estimator. It was decided to investigate a similar structure for synthesizing a dynamic controller with good robustness properties. The training architecture for such a neurocontrol synthesis is presented in Fig.1, where a multilayer feedforward net is trained to track the commanced trajectories given the vehicle outputs, the tracking errors, and the state vector of the 
vehicle model. (It is noted that providing the vehicle outputs and tracking errors to the neural network allows reconstruction of the commanded state). For each pilot selected trajectory $\bar{z}_{S E L}(t)$, a commanded trajectory $\bar{z}_{c}(t)$ is generated from (5). Prior to training, the dynamics of the pilot command filter, the actuators, and the vehicle model are discretized. The commanded trajectory $\bar{z}_{c}\left(t_{k}\right)$ and the vehicle state vector $\bar{x}\left(t_{k}\right)$ are scaled into $\bar{z}_{c}^{s}\left(t_{k}\right)$ and $\bar{x}^{2}\left(t_{k}\right)$ with scale factors that are representative of the vehicle dynamics over the range of pilot input commands. As shown in Fig.1, the control input to the actuators, $\bar{u}_{c}^{s}\left(t_{k}\right)$, and the control input to the plant, $\bar{u}_{a}^{s}\left(t_{k}\right)$, are normalized by their maximum value $\left(|W F|_{\text {max }},|\delta T V|_{\text {max }}\right)$. Due to the time-discretiration of the system dynamics, a commanded neurocontrol input generated at time $t_{k}$ will only affect the vehicle output at time $t_{k+2}$. During training, the weights increments at time $t_{k}$ are therefore calculated from $\bar{\epsilon}_{2}\left(t_{k}\right)=\bar{z}_{c}^{*}\left(t_{k+2}\right)-\bar{z}^{s}\left(t_{k}\right)$. This procedure ensures that, during training, the proper action will be commanded by the neurocontroller at time $t_{k}$ to achieve the desired tracking at time $t_{k+2}$.

In order to maximize the tracking performance while minimizing the costs associated with high control effort and high control rate requirements, the feedforward net of Fig. 1 is trained to minimize an objective function that is a weighted sum of tracking errors, and control input commands and rates $[4,5]$. The learning rate of backpropagation and the weights of the objective function (not to be confused with the weights of the neural network) are the "knobs" used by the control designer to "tune" the neurocontroller such that the design objectives are met. The commanded trajectories used for training were pitch rate doublets centered between $2.5 \mathrm{~s}$ and $5 \mathrm{~s}$ with a maximum absolute intensity of $0.5 \mathrm{in}$, and velocity step functions with a maximum absolute intensity of $20 \mathrm{ft} / \mathrm{sec}$ [5]. These types of commanded trajectories represent the frequency content of typical pilot command inputs. Towards the end of the training, the weights updates were calculated from the pitch-rate/velocity responses to such commands in the presence of an added time delay of $50 \mathrm{~ms}$ in both control channeis (location $\beta$ in Fig.2) in order to improve the phase stability margin of the neurocontroller. The network configuration in the training architecture of Fig. 1 has two hidden layers of 10 neurons, and the activation function of each neuron is given by the standard input/output response $y=\tanh (x)$.

Once trained, the two hidden-layer feedforward net is used in conjunction with a state estimator of the vehicle to command the control input to the actuator. In the closed-loop evaluation architecture of Fig.2, the state vector of the vehicie is estimated from a neural simulation of the dynamics of the nominal vehicle/actuators models. Given the vehicle output $\bar{z}\left(t_{k}\right)$, the tracking error $\bar{e}_{z}\left(t_{k}\right)=\tilde{z}_{c}\left(t_{k}\right)-\bar{z}\left(t_{k}\right)$, and an estimate $\hat{\bar{x}}\left(t_{k}\right)$ of the vehicle state vector, the feedforward net trained in Fig.1 is used to generate a control input $\bar{u}_{c}\left(t_{k}\right)$ as shown in Fig.2. The state estimate $\hat{\bar{x}}\left(t_{k}\right)$ and the control input $\bar{u}_{c}\left(t_{k}\right)$ are then passed to the neural estimator which estimates the next value of the vehicle state vector at time $t_{k}$. In Fig.2, the $A / D$ and $\mathrm{D} / \mathrm{A}$ converters are implemented through zero-order hold devices.

IV. Evaluation of the Dynamic Neurocontroller. The neurocontroller was evaluated in closed-loop on step pitch rate input commands, different from the doublets used in training. The input commands chosen to illustrate the neurocontrol performance were defined by the step pitch rate command $Q_{S E L}(t)=0.5$ in for $t \leq 3 \mathrm{sec}, Q_{S E L}(t)=0$ for $t>3 \mathrm{sec}$; applied simultaneously with the following step velocity command: $V_{S E L}(t>0)=20 \mathrm{ft} / \mathrm{sec}$. This type of input command was chosen to illustrate the system performance because it is quite demanding in that the pilot is commanding the aircraft to pitch up as well as accelerate to a higher veiocity.

Nominal Performance. As shown in Fig.3, the deviation from the ideal response is small for both pitch rate and velocity commands, while the control requirements $W F$ and $\delta T V$ are smooth. Also shown in Figs.3c \& $3 \mathrm{e}$ are the corresponding control and control rate requirements with the static neurocontroller of Ref.[5]. The nominal command tracking performance with the static neurocontroller was similar to that for the dynamic neurocontroller designed in this study, so the corresponding pitch-rate and velocity responses are not shown in Fig.3. Fig.3c indicates that the pitch rate command $\delta T V$ generated by the dynamic neurocontroller $\left(\delta T V_{N N \text { dynamac }}\right)$ is much smaller than the one generated by the static neurocontroller $\left(\delta T V_{N N}\right.$ static) Fig.3e shows that the control rate $\delta \dot{T} V_{N N}$ dynamic generated by the dynamic neurocontroller no longer rides the actuator rate limit, as it was the case for the static neurocontroller $\left(\delta \dot{T} V_{N N}\right.$ static $)$. For other classes of step velocity commands. e.g. $V_{S E L}(t)=-20 \mathrm{ft} / \mathrm{sec}$, the fuel flow requirement $W F$ of the dynamic neurocontroller is also reduced, no longer showing the oscillatory behavior as it had in the static neurocontrol design [5]. As wiil be demonstrated in the next subsections, the improved tracking-performance/control-effort tradeoff of the dynamic neurocontroller over the static neurocontroller $[5,8]$ enhances the robustness of the control in the presence of modelling uncertainties. Modelling uncertainties are due to neglected high order dynamics, parameter changes due to changes in flight conditions, and the margin of error associated with 
estimating model parameters based on analytical tools and experimental data. A classic specification for robustness, also used in the military specifications for design of flight control systems [7], is that of stability margins, specifically gain and phase margins [9].

Phase Margin. To estimate the phase robustness of the dynamic neurocontroller, the effect of the various time-delays encountered by the signals throughout the closed-loop system was modelled by introducing a delay $\tau_{d}$ between the actuators and the vehicle (location $\beta$ in the closed-loop evaluation architecture of Fig.2). The neurocontroller performance in tracking pitch rate and velocity commands is illustrated in Fig.4 for an added delay of $\tau_{d}=50 \mathrm{~ms}$ in the two control channels (WF and $\delta T V$ ). This value of $\tau_{d}$ is quite representative of the kinds of time delays to be expected in practical implementation of complex flight control designs. Fig. 4 also shows very little degradation of the tracking performance of the neurocontroller in the presence of such a time-delay, and it is to be noted here that the static neurocontroller of Ref.[5] resulted in a highly oscillatory pitch rate response with this value of $\tau_{d}$.

Gain Margin. To analyze the robustness of the neurocontroller to uncertainties of the type that can be modelled as gain changes at the plant output, closed-loop simulations were tried with various gain factors of the vehicle output measurements (location $\gamma$ in the closed-loop evaluation architecture of Fig.2). Closed-loop responses are illustrated in Fig.5 with a gain factor of 2 in both pitch rate and velocity measurements. It is noted that the gain margin of the handling qualities specifications [7] is between gain factors of 0.5 and 2 in each control channel. The performance of the neurocontroller in tracking pitch rate and velocity commands is very satisfactory in both cases of commands. Fig. 6 shows the pitch rate and velocity responses with a gain factor of 0.55 in pitch rate measurement, and a gain of 0.6 in velocity measurement. It is noted that, in closed-loop evaluation, the state vector of the vehicle is estimated by the neural estimator of the nominal vehicle model. It would therefore be interesting to test the possibility of increasing the gain margin of the neurocontroller within the handling qualities specifications by simulating vehicle modelling uncertainties in the neural estimator. As indicated in Fig.6, the neurocontroller is still able to stabilize the vehicle, yet with an expected loss of tracking performance, since the chosen pitch-rate/velocity command is quite demanding in terms of control effort and control rate requirements.

Error Loop Sensor Failure. In the classical approach of flight control design, an inner loop compensation $(\bar{z} \rightarrow \bar{u})$ is first designed to provide stability augmentation, and to place the augmented plant dynamics within the handling qualities specifications. An outer loop compensation $(\bar{e} \rightarrow \bar{u})$ is subsequently designed to provide decoupled command tracking in order to reduce pilot workload. The inner loop compensation of this dynamic neurocontroller was evaluated by considering failures in the outer compensation loops, i.e. failure in the error sensors (location $\alpha$ in the closed-loop evaluation architecture of Fig.2). The responses of the closed-loop system with failure in the $e_{Q}$ loop are shown in Fig.7. The neurocontroller very satisfactorily tracks the velocity command and provides stable response in pitch rate, whereas both velocity and pitch-rate responses with the static neurocontroller [5] were unstable in the presence of $e_{Q}$ loop failure. The closed-loop responses with failure in the $e_{V}$ loop are shown in Fig.8. The neurocontroller performance in tracking the commanded pitch rate is comparable to the tracking performance with the nominal vehicle model, and the velocity response is smooth. This indicates that the dynamic neurocontroller uses pitch rate and velocity measurements in a manner consistent with the classical idea of providing inner loop plant augmentation.

V. Conclusion. The synthesis of robust neurocontrollers for flight control was investigated via an aircraft control design example. The multivariable control design problem was set up as the task of following the trajectories generated from a model of the desired vehicle response dynamics to pilot command inputs. The neurocontroller has an internal structure consisting of a state-feedback neuro-regulator operating in conjunction with a neural estimator. This neurocontrol structure in conjunction with appropriate training allows for automatic generation of the neurocontroller dynamics that achieve the desired performance and robustness.

In contrast to the static neurocontroller [5], this dynamic neurocontroller achieves a satisfactory trackingperformance/control-effort tradeoff with good robustness properties in the presence of modelling uncertainties and error loop failures. The neurocontroller maintains performance and stability in the presence of timedelays and vehicle output gain variations that are to be expected in the practical implementations of complex fight control designs. Closed-loop simulations of the system also showed that the neurocontroller could track the velocity command when the sensor measuring the tracking error of the pitch rate command would fail, and vice-versa. The structure of the neurocontroller, which provides stability augmentation and decoupled command tracking, is therefore consistent with the classical approach of flight control design. 


\section{References.}

[1] Special Sections on Neural Networks for Control Systems, IEEE Control System Magazine, Vol.9, No.3, pp. 25-59, April 1989; and Vol.10, No.3, pp. 3-87, April 1990.

[2] Narendra, K. S., and Parthasarathy, K., "Identification and Control of Dynamical Systems Using Neural Networks", IEEE Trans. on Neurai Networks, Vol.1, No.1, March 1990.

[3] White, D., and Sofge, D., "Neural Network Based Process Optimization and Control", 29th IEEE Conference on Decision and Control, Honolulu, HI, Dec. 1990.

[4] Troudet, T.. Garg, S., Mattern, D. L., and Merrill, W., "Towards Practical Control Design Using Neural Computation", Internationai Joint Conference on Neural Networks, Seattle, WA, July 1991.

[5] Troudet, T., Garg, S., and Merrill, W., "Neural Network Application to Aircreft Control System Design", AIAA Guidance, Navigation and Control Conference, New Orleans, LA, Aug. 1991.

[6] Garg, S., Mattern, D.L., and Bullard, R.E.,"Integrated Flight/Propuision Control System Design Based on a Centralized Approach", Journal of Guidance, Control and Dynamics, Vol.14, No.1, Jan.-Feb. 1991.

[7] "Military Specification - Flying Qualities of Piloted Airplanes", MIL-F-8785C, USAF, WPAFB, OH, Nov. 1980.

[8] Troudet, T., Garg, S., and Merrill, W., "Neurocontrol Design and Analysis for a Multivariable Aircraft Control Problem", submitted to Computers and Mathematics with Applications, Sep. 1991.

[9] Ogata, K., "Modern Control Engineering", Prentice Hall Inc., 1970.
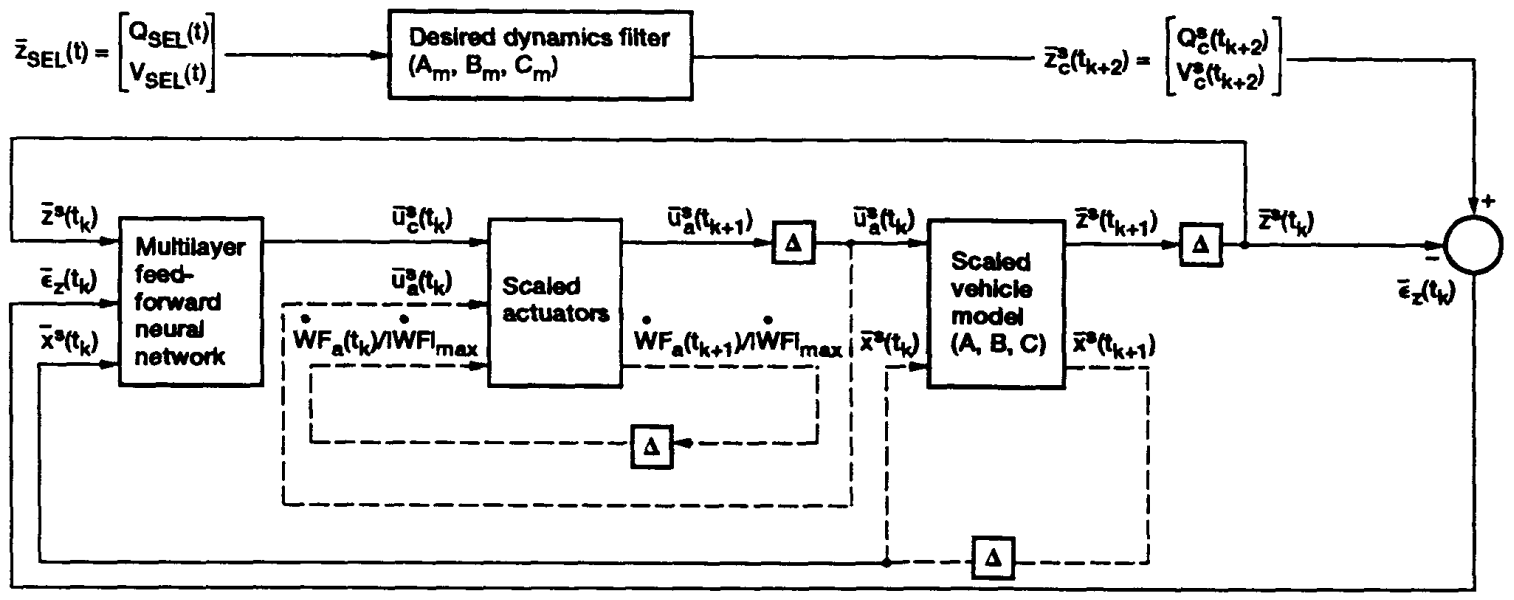

Figure 1.-Training architecture.

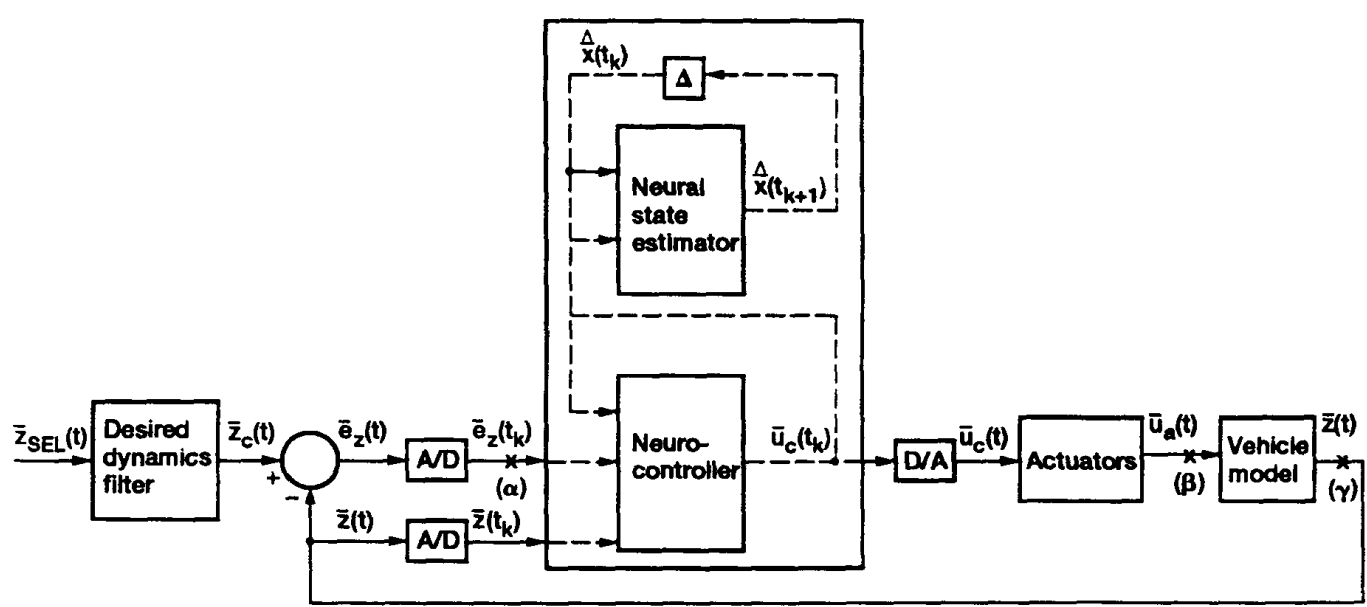

Figure 2.-Closed-loop evaluation architecture of the dymamic neurocontroller. 


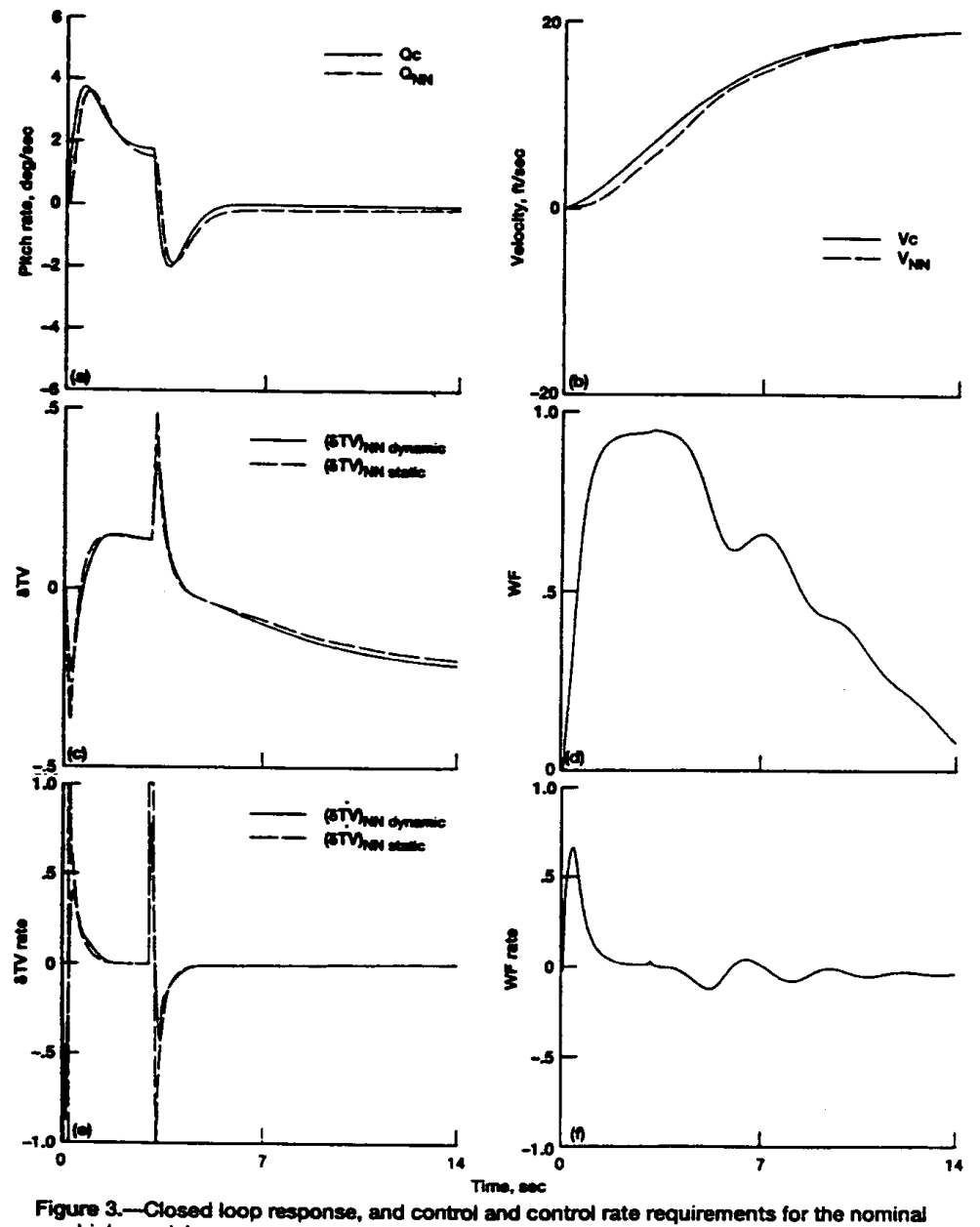
Figure 3.-Closed loop response, and control and control rate requirements for the nomina
vehicie model.

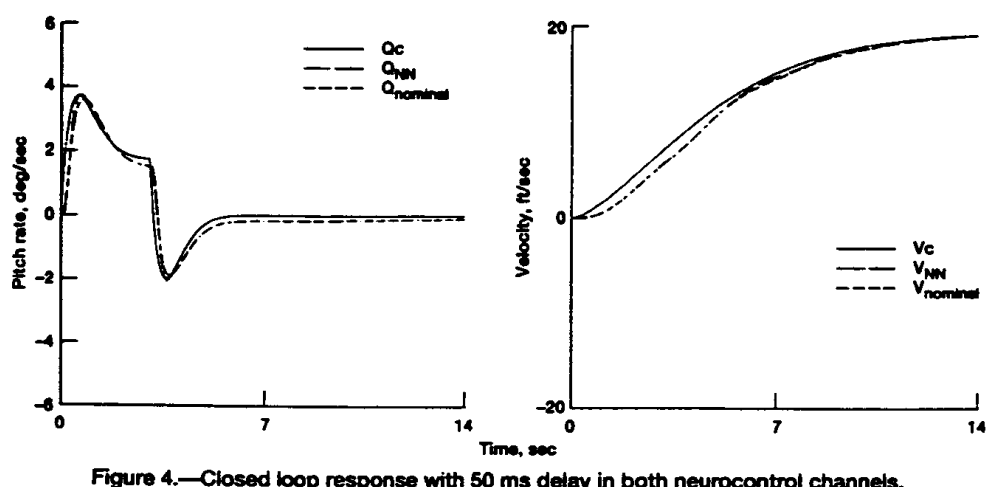



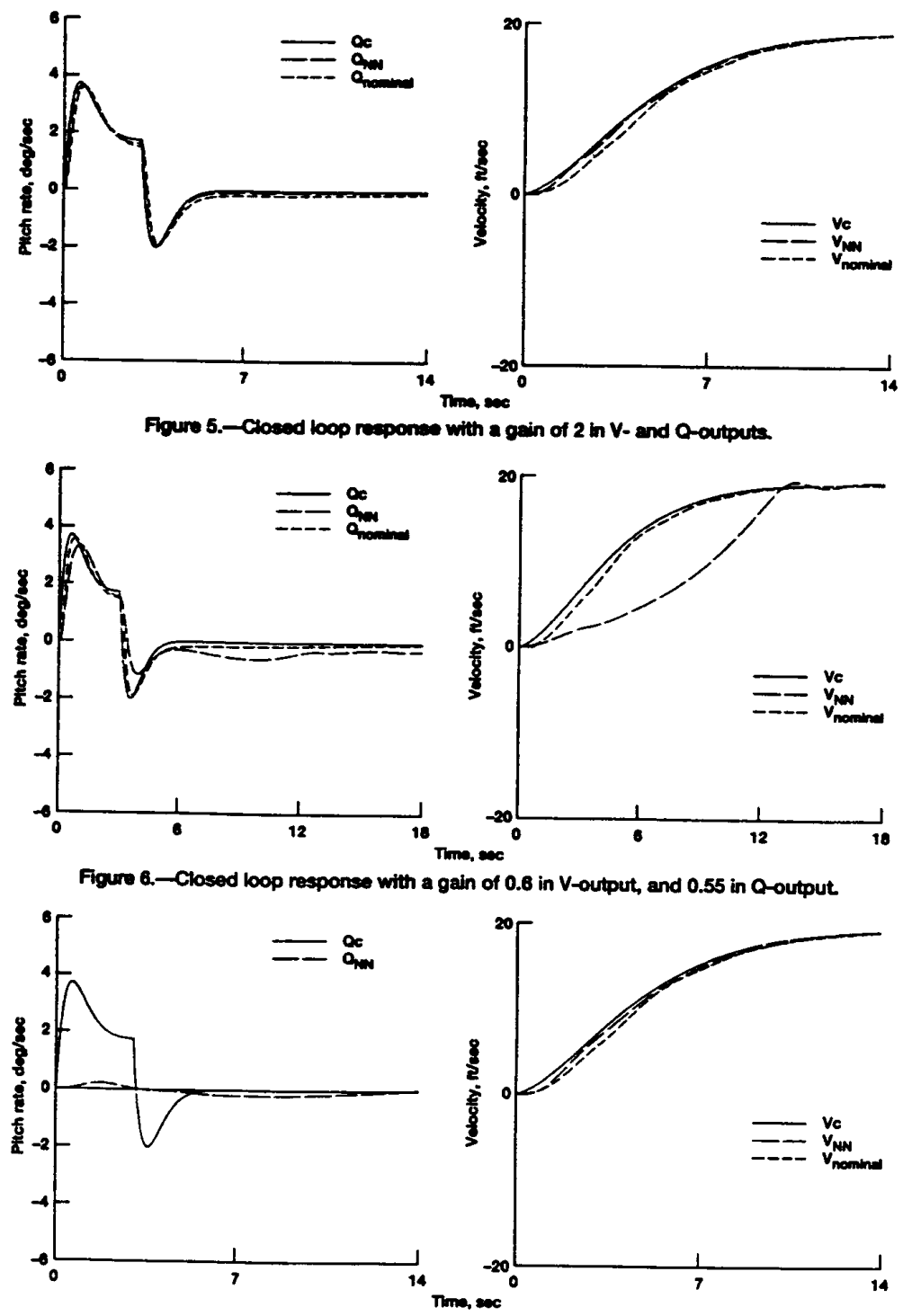

Figure 7. -Closed loop response with en sensor failure.

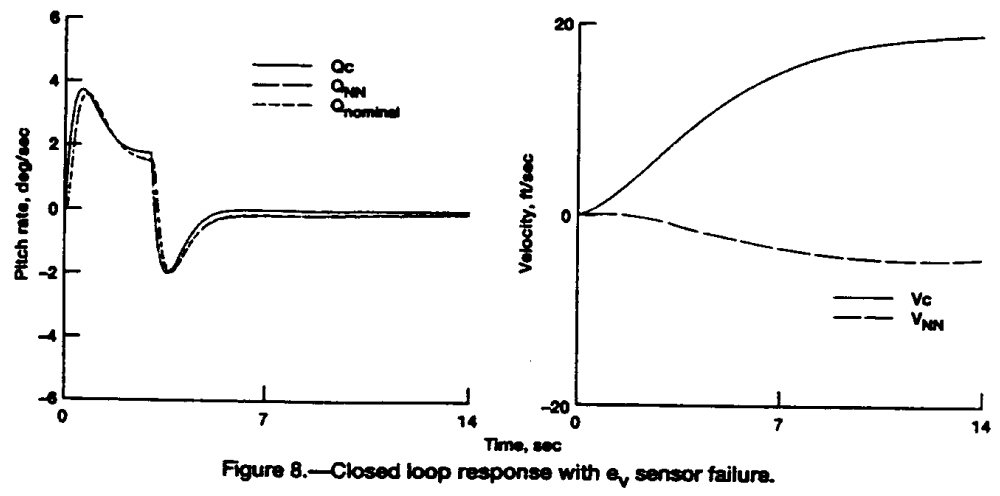

I-314 